\title{
ACS Chemical Biology
}

This document is confidential and is proprietary to the American Chemical Society and its authors. Do not copy or disclose without written permission. If you have received this item in error, notify the sender and delete all copies.

\section{Unique DC-SIGN clustering activity of a small glycomimetic: a lesson for ligand design}

\begin{tabular}{|r|l|}
\hline Journal: & ACS Chemical Biology \\
\hline Manuscript ID: & cb-2014-00054h \\
\hline Manuscript Type: & Article \\
\hline Date Submitted by the Author: & 24-Jan-2014 \\
\hline Complete List of Authors: & $\begin{array}{l}\text { Sutkeviciute, Ieva; Univ. Grenoble Alpes, Institut de Biologie Structurale } \\
\text { Thépaut, Michel; CNRS, Institut de Biologie Structurale } \\
\text { Sattin, Sara; Universita di Milano, Departimento di Chimica } \\
\text { Berzi, Angela; Universita' di Milano, Department of Biomedical and Clinical } \\
\text { Sciences } \\
\text { McGeagh, John; Anterio Consult\&Research, } \\
\text { Grudinin, Sergei; INRIA, NANO-D } \\
\text { Weiser, Jörg; Anterio Consult \& Research GmbH, } \\
\text { Le Roy, Aline; University Joseph Fourier, Institut de Biologie Structurale } \\
\text { Reina, Jose; CSIC, Instituto de Investigaciones Quimicas } \\
\text { Rojo, Javier; CSIC, Instituto de Investigaciones Quimicas } \\
\text { Clerici, Mario; Universita' di Milano, Department of Biomedical and Clinical } \\
\text { Sciences } \\
\text { Bernardi, Anna; Universita' di Milano, Chimica Organica e Industriale } \\
\text { Ebel, Christine; CNRS, Institut de Biologie Structurale } \\
\text { Fieschi, Franck; Université Joseph Fourier, Institut de Biologie Structurale }\end{array}$ \\
\hline
\end{tabular}

\section{SCHOLARONE"}

Manuscripts 


\title{
Unique DC-SIGN clustering activity of a small glycomimetic: a lesson for ligand design
}

\author{
Ieva Sutkeviciute ${ }^{1,2,3}$, Michel Thépaut ${ }^{1,2,3}$, Sara Sattin ${ }^{4}$, Angela Berzi ${ }^{5}$, John McGeagh $^{6}$, Sergei \\ Grudinin $^{7,8}$, Jörg Weiser ${ }^{6}$, Aline Le Roy ${ }^{1,2,3}$, Jose J. Reina ${ }^{9}$, Javier Rojo ${ }^{9}$, Mario Clerici ${ }^{10}$, Anna \\ Bernardi $^{4}$, Christine Ebel ${ }^{1,2,3}$, Franck Fieschi ${ }^{1,2,3^{*}}$
}

\begin{abstract}
${ }^{1}$ Univ. Grenoble Alpes, Institut de Biologie Structurale (IBS), Grenoble, F-38027, France; ${ }^{2}$ CNRS, IBS Grenoble, F-38000, France; ${ }^{3}$ CEA, DSV-IBS, Grenoble, F-38000, France; 4 Universita' di Milano, Dipartimento di Chimica, via Golgi 19, 20133 Milano - Italy; 5Department of Biomedical and Clinical Sciences, University of Milan, 20157 Milan, Italy; ${ }^{6}$ Anterio Consult\&Research GmbH, Augustaanlage 23, D68165 Mannheim-Germany; ${ }^{7}$ INRIA Grenoble, Saint Ismier Cedex F-38334, France; ${ }^{8}$ CNRS Laboratoire Jean Kuntzmann, 38041 Grenoble, France; ${ }^{9}$ Glycosystems Laboratory, Instituto de Investigaciones Químicas (IIQ), CSIC - Universidad de Sevilla, Av. Américo Vespucio 49, Seville 41092 Spain; ${ }^{10}$ Department of Physiopatology and Transplantation, University of Milan, Milan, and Don C. Gnocchi Foundation ONLUS, IRCCS, 20148 Milan, Italy.
\end{abstract}

*Corresponding author. E-mail: fieschi@ibs.fr; Tel: +33-(0)-4-57 428523. 


\begin{abstract}
DC-SIGN is a dendritic cell-specific C-type lectin receptor that recognizes highly glycosylated ligands expressed on the surface of various pathogens. This receptor plays an important role in the early stages of many viral infections, including HIV, which makes it an interesting therapeutic target. Glycomimetic compounds are good drug candidates for DC-SIGN inhibition due to their high solubility, resistance to glycosidases and non-toxicity. We studied the structural properties of the interaction of the tetrameric DC-SIGN extracellular domain (ECD), with two glycomimetic antagonists, a pseudo-mannobioside (1) and a linear pseudo-mannotrioside (2). Though the inhibitory potency of 2, as measured by SPR competition experiments, was one order of magnitude higher than that of $\mathbf{1}$, crystal structures of the complexes within DC-SIGN carbohydrate recognition domain showed the same binding mode for both compounds. Moreover, when coupled to multivalent scaffolds, the inhibitory potencies of these compounds became uniform. Combining isothermal titration microcalorimetry, analytical ultracentrifugation and dynamic light scattering techniques to study DC-SIGN ECD interaction with these glycomimetics, revealed that $\mathbf{2}$ is able, without any multivalent presentation, to cluster DC-SIGN tetramers leading to an artificially overestimated inhibitory potency. The use of multivalent scaffolds presenting $\mathbf{1}$ or $\mathbf{2}$ in HIV trans-infection inhibition assay confirms the loss of potency of $\mathbf{2}$ upon coupling and the equal efficacy of chemically simpler compound $\mathbf{1}$. This study documents a unique case where, among two active compounds chemically derived, the compound with the lower apparent activity is the optimal lead for further drug development.
\end{abstract}

Keywords: C-Type Lectin, HIV, glycomimetics, clustering, analytical ultracentrifugation, isothermal microcalorimetry 


\section{Introduction}

Immature dendritic cells (DCs) are professional antigen presenting cells of the innate immunity. They routinely survey peripheral tissues for invading pathogens, and present the antigens to the $\mathrm{T}$ cells thus boosting pathogen-specific adaptive immune responses(1). Pattern recognition receptors (PRRs) of DCs are instrumental in capturing pathogens through the recognition of pathogen associated molecular patterns (PAMPs). DCs express a wide range of PRRs including toll-like receptors (TLRs) and C-type lectin receptors (CLRs), enabling DCs to recognize the majority of invading pathogens $(2,3)$. One of these PRRs is DC-SIGN (Dendritic Cell-Specific ICAM-3 Grabbing Non-integrin), a CLR abundantly expressed on immature DCs that has been extensively studied because of its intriguing roles in immunity(4, 5). DC-SIGN was recognized as a receptor hijacked by various dangerous pathogens, including viruses like HIV and Ebola, bacteria, fungi and parasites, to evade or modulate host immune responses enhancing their infectivity $(6,7)$. These findings highlighted DC-SIGN as an interesting therapeutic target and inspired many research groups to attempt the design of antagonists for infection prevention.

DC-SIGN is a type II membrane protein with a cytosolic domain followed by a transmembrane region and an extracellular domain (ECD). The latter is organized into an elongated neck region and a globular C-terminal carbohydrate recognition domain (CRD), which binds fucose and mannosecontaining oligosaccharides in a calcium-dependent manner $(8,9)$. The neck drives lectin's oligomerization into tetramers and serves as a stalk raising CRDs well above the cell membrane $(320 \AA)$ and presenting them in a tetravalent manner with $\sim 40 \AA$ distances between vicinal binding sites (10). These tetramers are further clustered to microdomains at the cell membrane $(11,12)$.

Although monosaccharide affinity to DC-SIGN is very low, multimeric DC-SIGN organization together with clustered presentation of pathogen glycans leads to the affinity enhancement through avidity effects. Hence, the strategies to develop good DC-SIGN inhibitors not only involve optimization of the monovalent ligands, but also include extensive search for the proper scaffolds for multimeric ligand presentation, thus ensuring efficient competition with highly multivalent PAMPs (reviewed in $(13,14))$.

Our groups focus on the development of fucose- and mannose-based glycomimetic inhibitors of DC-SIGN. We recently published series of LewisX trisaccharide mimics, which had slightly better affinity as compared to natural LewisX and an improved selectivity to DC-SIGN versus langerin(15), a 
C-type lectin implicated in the protection from HIV infection(16). We have also developed two mannose-type glycomimetics, corresponding to a terminal branch of high-mannose glycan, which are the target ligands for DC-SIGN on the gp120 HIV envelope protein. Thus, we produced a Man $\alpha 1$ -

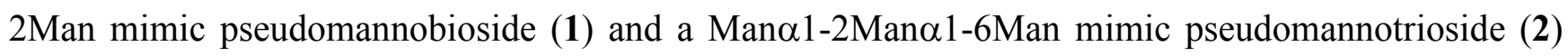
and evaluated them as DC-SIGN inhibitors. The initial surface plasmon resonance (SPR) competition assay indicated that $\mathbf{2}$ had an order of magnitude lower $\mathrm{IC}_{50}$ than 1(17). Hence, this compound was selected to generate a tetravalent dendron 3.2, which in turn was tested for the ability to inhibit HIV trans-infection of T-lymphocytes mediated by B-THP-1/DC-SIGN cells(17) as well as HIV transmission inhibition in cervical tissue explants(18). Indeed, the dendron $\mathbf{3 . 2}$ displayed a very promising anti-HIV activity in these assays and was demonstrated to be non-toxic.

Additionally, the third generation (G3) Boltorn type dendrimers 4 bearing an average of 30-32 copies of 1 (compound 4.1) or 2 (compound 4.2) were built and evaluated in SPR competition experiments and DC-SIGN-mediated Ebola infection assays(19). Both dendrimers were highly active in inhibiting Ebola infection, but surprisingly, no significant difference was found in their potencies ( $\mathrm{IC}_{50} \sim 20 \mathrm{nM}$ for both compounds). The SPR competition experiments gave a similar outcome (Figure 1): while the activities of the monovalent compounds have almost one order of magnitude difference in favor of 2, nearly the same affinities were observed for tetravalent (3.1 and 3.2) and multivalent (4.1 and 4.2) forms of 1 and 2. Furthermore, the monovalent and tetravalent forms of 2 ( 2 and 3.2) had basically the same activities. 
Recently, we have structurally characterized the binding of $\mathbf{1}$ to DC-SIGN CRD(20). X-ray crystallography of co-crystallized DC-SIGN CRD/1 complex revealed an unexpected binding mode of the molecule, which was confirmed by solution studies using transfer NOE (tr-NOESY) and saturation transfer difference (STD) NMR experiments. Although 1 and its natural counterpart Mano1-2Man have similar affinities for DC-SIGN as observed in SPR competition test $\left(\mathrm{IC}_{50}\right.$ values $1.0 \mathrm{mM}$ and $0.9 \mathrm{mM}$, respectively), our results indicated that $\mathbf{1}$, contrary to Mand1-2Man, has only a single binding mode within DC-SIGN CRD, a prerequisite for a good lead compound for further chemical modifications to improve affinity and selectivity. We also found that $\mathbf{1}$ has an enhanced selectivity towards DC-SIGN compared to langerin. This is an important feature for the development of microbicides that shouldn't interfere with the protective function of langerin, especially due to its expression on Langerhans cells, which constitute the very first barrier for invading HIV virions in genital and rectal mucosa(16).

Herein we present our efforts to unravel the underlying reasons for the above-described surprising behavior of the compound $\mathbf{2}$. Through series of biophysical studies we found that this small glycomimetic, to our surprise, acts as a bivalent molecule, and this peculiar property led to an artificial 
overestimation of its potency in competition assays where soluble DC-SIGN tetramers are used. This work emphasizes the importance of thorough and critical structural investigation of leads from primary screening campaigns and highlights specific design challenges for the discovery of potent antagonists of lectins' activity.

\section{Results and discussion}

The study described in this paper was motivated by the need to understand why the relatively high efficiency of $\mathbf{2}$ is lost when the compound is tethered on multivalent scaffolds, an essential information in order to shape our ligand design program. We have co-crystallized the latter glycomimetic with DC-SIGN CRD and solved the X-ray structure. Furthermore, we investigated the thermodynamic and hydrodynamic properties of DC-SIGN ECD interaction with $\mathbf{1}$ and $\mathbf{2}$ by isothermal titration microcalorimetry (ITC) and analytical ultracentrifugation (AUC). Our results indicated that monovalent 2 in fact functions as a bivalent molecule capable of bridging DC-SIGN tetramers, a very peculiar property for such a rather small molecule with relatively not so high affinity. Such peculiar property of $\mathbf{2}$ resulted in an artificial overestimation of its potency in competition assays where soluble DC-SIGN tetramers are used.

\section{The X-ray structure of DC-SIGN CRD/2 complex and its comparison with DC-SIGN CRD/1}

In order to compare the binding modes of $\mathbf{1}$ and $\mathbf{2}$ within DC-SIGN and thus to shed light on the underlying reasons for the binding affinity of mono- and polyvalent versions of $\mathbf{2}$, we co-crystallized $\mathbf{2}$ with monomeric DC-SIGN CRD and solved the X-ray crystal structure.

Similarly to the recently published structure of DC-SIGN/1, the crystals of 2 in complex with DC-SIGN CRD also had a single copy of CRD in an asymmetric unit with $\mathrm{P} 4_{3} 2{ }_{1} 2$ space group. The structure was solved by molecular replacement, at 1.35 Å resolution (see Table S1 in SI). 

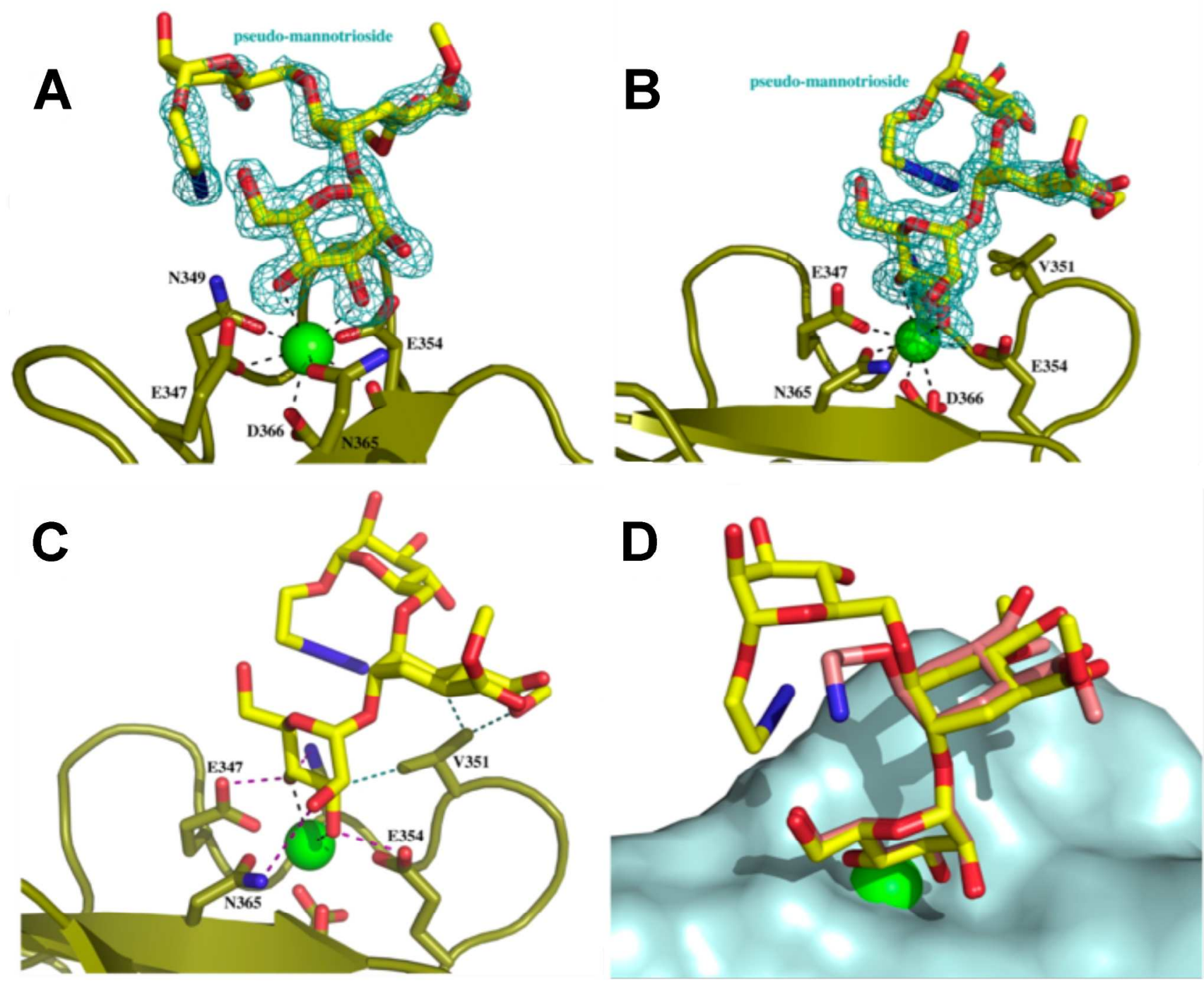

Figure 2. The binding mode of pseudo-mannotrioside 2 within DC-SIGN CRD. (A) and (B) Two orientations of the bound ligand superimposed with the Fo-Fc electron density map (cyan $3 \sigma$ contour); the protein carbon backbone is represented in olive cartoon with amino acids contributing to binding highlighted by stick representation; 2 is shown in yellow sticks. V351 exhibits two alternative conformations with 50\% occupancy in the crystal structure, both of them are represented in (B). (C) pseudo-mannotrioside interaction to DC-SIGN. Hydrogen bonds are shown as dashed purple lines, Ca ${ }^{2+}$ coordination bonds are dashed black lines, and key van der Waals interaction are indicated by dashed blue lines. (D) The superimposition of $\mathbf{1}$ (pink sticks) and $\mathbf{2}$ (yellow sticks) crystal structures bound to DC-SIGN CRD (pale cyan surface representation). Oxygen and nitrogen atoms are in red and blue, $\mathrm{Ca}^{2+}$ ion is a green sphere.

When the structure was solved using the model without sugar, an electron density was observed on $\mathrm{Ca}^{2+}$ ion in the canonical carbohydrate binding site, indicating the presence of pseudomannotrioside bound to DC-SIGN through a conventional $\mathrm{Ca}^{2+}$-coordination by the 3-OH and 4-OH groups of the non-reducing end mannose moiety (Figure $2 \mathrm{~A}$ and $2 \mathrm{~B}$ ). It appeared that the binding mode of 2 within DC-SIGN CRD (Figure 2A) was the same as that of 1, with exactly the same orientation of the common portion of the molecule (Figure 2C and 2D). To our surprise, no additional contacts (other 
than those described previously for $1, \mathrm{pdb} 2 \mathrm{xr} 5$ ) with the protein were observed. While the nonreducing mannose moiety made coordination bonds with $\mathrm{Ca}^{2+}$ ion with its 3-OH and 4-OH groups and C6 methylene of the cyclohexane ring retained the van der Waals contact with Val351 side chain of the protein, the reducing-end mannose was oriented away from the protein and apparently didn't make any contacts. Moreover, the electron densities of this second mannose unit as well as of its ethylazide linker were rather poorly defined, suggesting a higher flexibility of these parts (Figure 2A). Thus the revealed structure of $\mathbf{2}$ within DC-SIGN didn't provide any clue about the reason why it could be a better ligand than 1 for DC-SIGN having apparently the same binding mode and contacts with the protein. This intriguing finding prompted us to investigate the interaction further on.

\section{Thermodynamic characterization of DC-SIGN interaction with 1 and 2}

To evaluate whether positive entropy contributions were responsible for the higher affinity of monovalent 2 relative to $\mathbf{1}$, we analyzed the interaction of both glycomimetics with tetrameric DCSIGN by ITC. At the first set, the same experimental conditions with identical concentrations of interaction components were used. The pseudosaccharides $(12.7 \mathrm{mM})$ were titrated into lectin solution (71 $\mu \mathrm{M}$ with respect to binding sites).
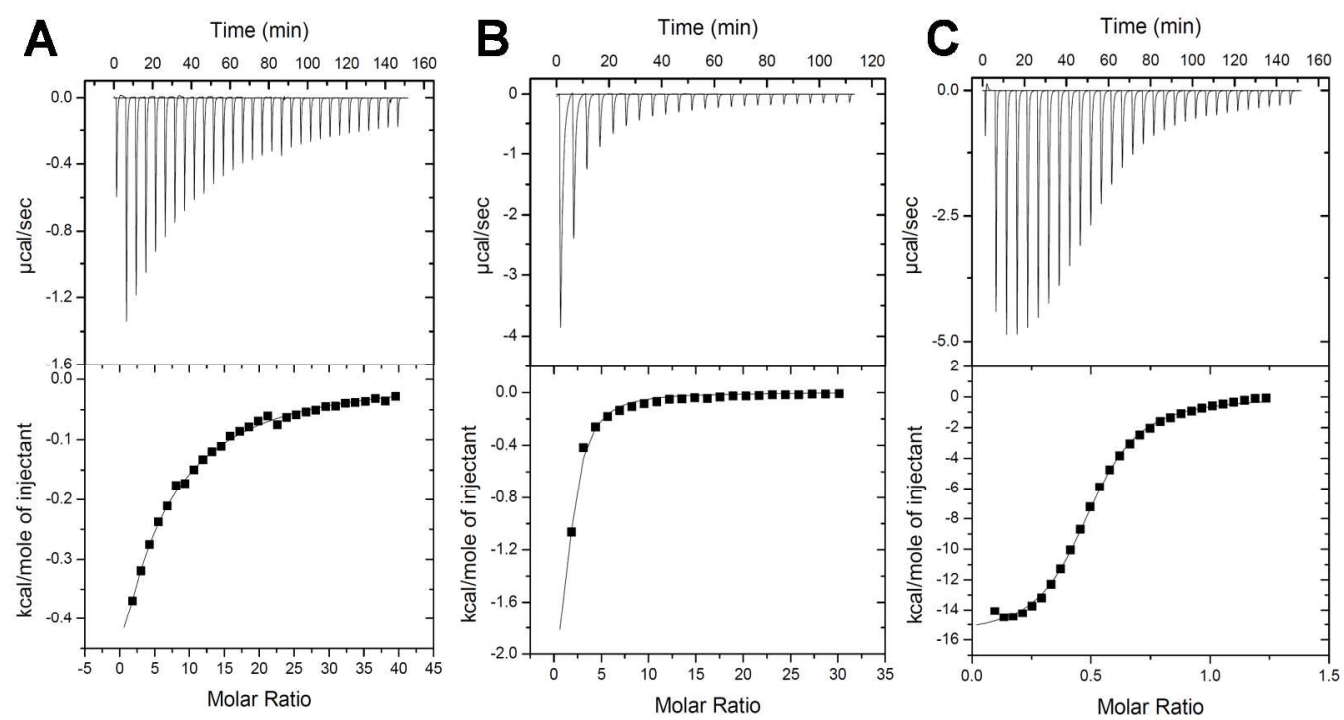

Figure 3. ITC results of $\mathbf{1}$ and $\mathbf{2}$ titrations to DC-SIGN ECD. (A) and (B) show titrations of $\mathbf{1}$ and $\mathbf{2}$, respectively, at $12.7 \mathrm{mM}$ to DC-SIGN ECD $(71 \mu \mathrm{M})$. (C) $2(1.18 \mathrm{mM})$ titration to $214 \mu \mathrm{M}$ DC-SIGN ECD. Upper panels show the titration thermograms and lower panels the data integration with fitted curves (1:1 binding model).

The ITC data (Figure 3A and 3B) indicated the low affinity of the ligands as the titration curves didn't adopt the full sigmoidal shape. Fitting one binding site model to the data with an assumed stoichiometry value $n$ fixed to 1 yielded the $K_{\mathrm{D}}$ of $990.10 \pm 19.7 \mu \mathrm{M}$ and $75.76 \pm 7.29 \mu \mathrm{M}$ for $\mathbf{1}$ and $\mathbf{2}$, 
respectively, but the low affinity prevented reliable interaction enthalpies and entropies to be obtained and compared(21). Nevertheless, an intriguing outcome could be observed: the first injections of $\mathbf{2}$ to DC-SIGN solution resulted in twice more heat released using the same concentrations of both ligands and the receptor. This suggested a higher affinity of $\mathbf{2}$ than $\mathbf{1}$, which could not be explained by the Xray data since $\mathbf{2}$ didn't seem to make any other additional contacts compared to $\mathbf{1}$.

Because $\mathbf{2}$ had apparently higher affinity to DC-SIGN, we repeated the titration with different concentrations (1.18 mM of 2 titrated to $214 \mu \mathrm{M}$ of DC-SIGN ECD) in order to perform it in a more relevant concentration range (Figure 3C). Fitting the same model this time gave $K_{\mathrm{D}}$ of $5.26 \pm 0.29 \mu \mathrm{M}$, $\Delta H=-15.80 \pm 0.15 \mathrm{kcal} \mathrm{mol}^{-1}, T \Delta S=-8.57 \mathrm{kcal} \mathrm{mol}^{-1}$, and $\Delta G$ of $-7.20 \mathrm{kcal} \mathrm{mol}^{-1}$. Thus a difference of 2 orders of magnitude between the $K_{\mathrm{D}}$ values of 1 and 2 was determined $(990 \mu \mathrm{M}$ and $5 \mu \mathrm{M}$, respectively), which is much higher than what could be initially expected from the SPR data and highlights even a stronger difference in the activities of the two molecules.

Furthermore, fitting of the data in Figure 3C yielded $n=0.5$ stoichiometry. Because additional DC-SIGN ECD purification step has been applied for this experiment (SI Figure S2), the contribution of non-active binding sites to $n<1$ value was excluded. Interaction with $n=0.5$ in this case means that two DC-SIGN CRDs share one molecule 2, or in other words, two DC-SIGN tetramers bind four copies of $\mathbf{2}$, on average. Since $\mathbf{2}$ is a relatively small molecule, it is impossible for it to reach two different CRDs within the same tetramer (the approximate distance between vicinal binding sites within the tetramer is $40 \AA(10))$. Hence, this stoichiometry suggests that the same molecule of 2 bridges two DC-SIGN tetramers by simultaneously binding to one CRD in each, and would explain why under the same experimental conditions titrating DC-SIGN with 2 released markedly more heat than titrating with 1.

\section{Analytical Ultracentrifugation analysis of DC-SIGN/1 and DC-SIGN/2 complexes}

To check the hypothesis that 2 can bridge DC-SIGN tetramers, sedimentation velocity (SV) experiments were performed on the samples retrieved from the ITC measurements, where the ligands were in 26-fold excess relative to the lectin binding sites. Figure 4 compares the sedimentation profiles registered at $42000 \mathrm{rpm}$ at the same time intervals $(1 \mathrm{~h})$ for each of the three samples (pure protein, Figure 4A; DC-SIGN+1, Figure 4B; DC-SIGN+2, Figure 4C). Clearly, DC-SIGN alone or incubated with 1 sediments similarly, while DC-SIGN incubated with 2 moves faster, suggesting that the association of DC-SIGN tetramers is induced by $\mathbf{2}$ but not by $\mathbf{1}$. 

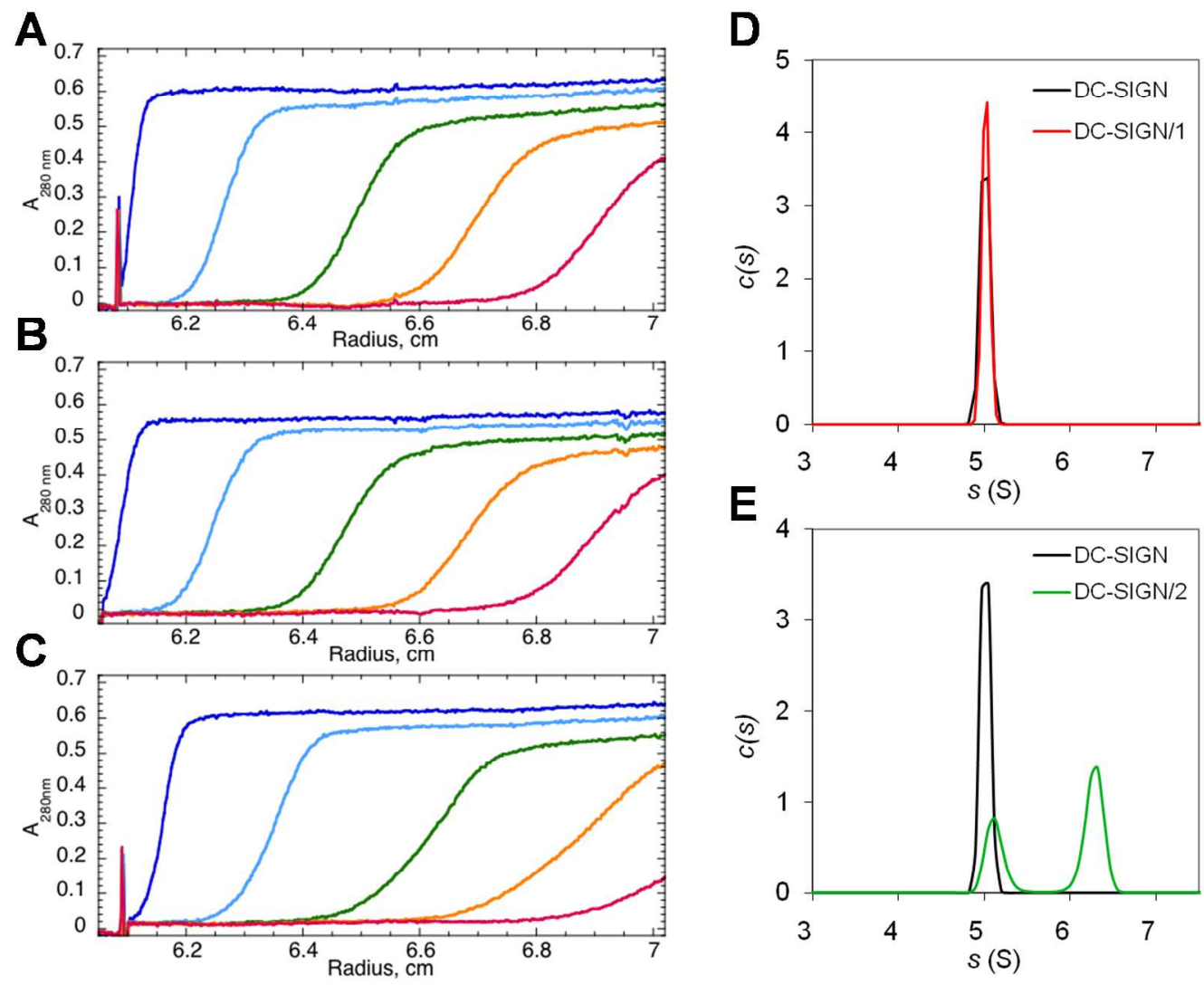

Figure 4. SV experiments of $62 \mu \mathrm{M}$ DC-SIGN ECD alone and in presence of 1 or 2 at $1.63 \mathrm{mM}$. Experimental data showing concentration profiles of DC-SIGN alone (A), in the presence of 1 (B) and 2 (C) recorded with time intervals between successive profiles of $40 \mathrm{~min}$ for the two first ones, then $55 \mathrm{~min}$; the first profiles at panels (A), (B) and (C) were obtained 4, 10 and 14 min, respectively, after the beginning of the centrifugation. (D) and (E) show the superimposition of the $c(s)$ curves for DC-SIGN alone and with $1(\mathrm{D})$ and $\mathbf{2}(\mathrm{E})$.

The data were treated in terms of size distribution analysis. Figure 4D shows a nearly perfect superposition of the $c(s)$ distributions obtained with DC-SIGN alone and in the presence of $\mathbf{1}$. On the other hand, glycomimetic 2 induced the formation of larger species (Figure 4E).

DC-SIGN alone and with 1 sediments at $s_{20 \mathrm{w}}=5.2 \mathrm{~S}$, close to $s_{20 \mathrm{w}}=5.4 \mathrm{~S}$ previously published for the tetramer at infinite dilution $(10,22)$. The additional peak observed for DC-SIGN in the presence of 2 is at $s_{20 \mathrm{w}}=6.5 \mathrm{~S}$. This value may correspond to a more compact tetramer $\left(f / f_{\min }=1.6\right.$ instead of 1.9 for DC-SIGN alone) or to a more elongated complex of two tetramers $\left(f / f_{\min }=2.5\right)$. However, this experiment was done only at one ratio, and we cannot exclude that $6.5 \mathrm{~S}$ peak corresponds to an intermediate value between the $s$-value of the tetramer and of the larger complex. The shape of the sedimentation boundary, thus the $c(s)$, indeed depends on the kinetics and thermodynamics of the interaction. 
To elucidate the origin of these larger species, a second set of SV experiments was performed using several 2/DC-SIGN molar ratios $(0,0.25,0.5,1,5,11$ and 27$)$ and the results are summarized in Figure 5.
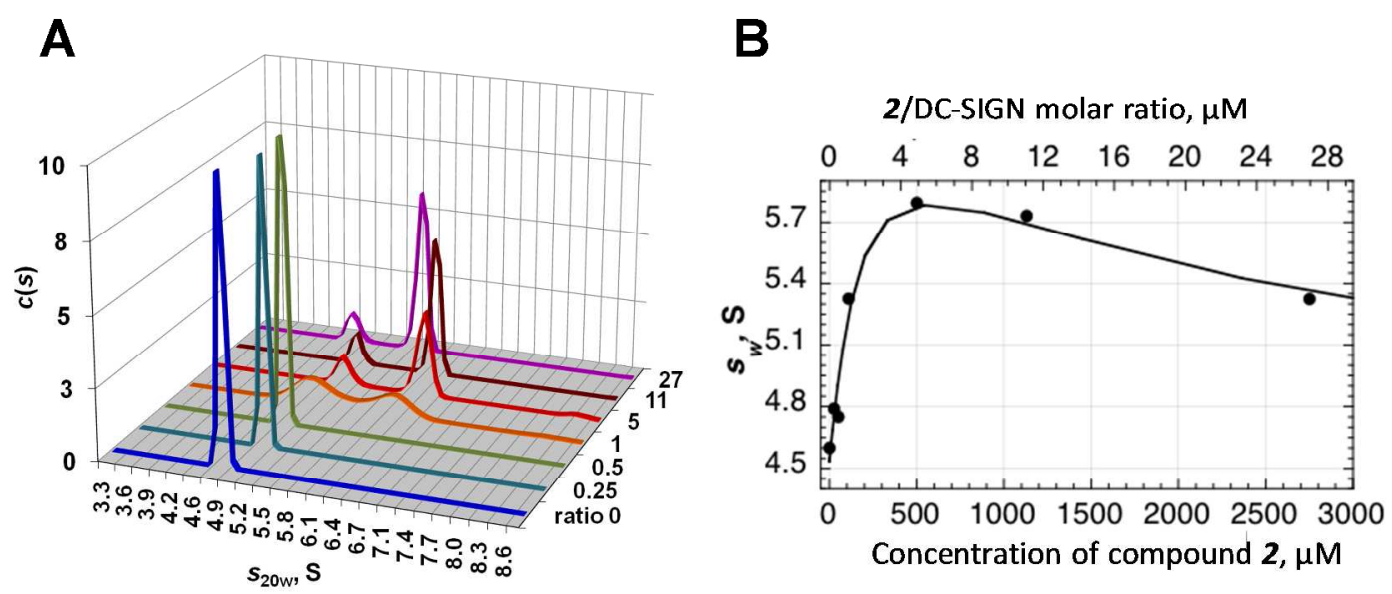

Figure 5. SV experiments of DC-SIGN ECD alone and in the presence of 2 at different 2 /DC-SIGN molar ratios. (A) The overlaid $c(s)$ curves for DC-SIGN ECD alone and with $\mathbf{2}$ at different ratios. (B) The evolution of $s_{w}$-values as a function of 2 concentration. DC-SIGN ECD concentration was $102 \mu \mathrm{M}$ with respect to binding sites.

DC-SIGN ECD alone sediments at $s_{20 \mathrm{w}}=4.8 \mathrm{~S}$, which is a lower value than measured with DC-SIGN ECD at $62 \mu \mathrm{M}$. This difference may be related to excluded volume effects, which are expected at this concentration $\left(4 \mathrm{mg} \mathrm{mL}^{-1}\right)$ considering the elongated shape of DC-SIGN ECD. The $s$ value of this peak is slightly increasing up to a 2/DC-SIGN ECD ratio of 0.5 (Figure 5A). Starting from ratio 1 , an additional peak emerges at approximately $s_{20 \mathrm{w}}=6.3 \mathrm{~S}$, corresponding to the formation of larger species. Increasing the concentration of $\mathbf{2}$, the proportion of this peak increases up to $80 \%$ of the total signal at a $2 / \mathrm{DC}-\mathrm{SIGN}$ ECD ratio of 11 . The $s$-values of both peaks start to decrease slightly from ratio 5: for the largest species the observed $s_{20 \mathrm{w}}$ maximum is $6.3 \mathrm{~S}$ at ratio 5 , which decreases to $5.7 \mathrm{~S}$ at ratio 27; for the smallest species at the same ratios the corresponding $s_{20 \mathrm{w}}$-values are 5.2 and $4.7 \mathrm{~S}$. These variations of the absolute $s_{20 \mathrm{w}}$-values are likely not related to experimental errors, but reflect the complexity of a reaction boundary: for fast reactions, indeed, association-dissociation processes affect the sedimentation boundary $(23,24)$. The integration of each $c(s)$ curve under the two peaks gives, on one hand, the total absorbance reflecting DC-SIGN ECD concentration (similar at all ratios), and on the other hand, the weight-average sedimentation coefficient value $\left(s_{w}\right)$ that does not depend on the interaction kinetics. We attempted the $s_{w}$-isotherm analysis using SEDPHAT, but fitting heteroassociation models failed, most likely due to the presence of a more complicated system and to nonideality conditions. Nevertheless, the data analysis in terms of $s_{w}$-value (Figure 5B) clearly shows that 
the addition of 2 up to $500 \mu \mathrm{M}$ concentration to $102 \mu \mathrm{M}$ DC-SIGN solution participates in the formation of higher macromolecular complexes as indicated by the increase of $s_{w}$-value, while further excess of the compound induces the disassembly of DC-SIGN complexes. Although this type of data analysis can be used to extract the interaction affinities $(23,24)$, the studied system is apparently too complicated (tetravalent protein, presumably bivalent ligand, excluded volume effects), therefore no model could be fitted to the data.

\section{Characterization of DC-SIGN/2 interaction by static and dynamic light scattering}

DC-SIGN association, induced by 2, was also examined by Static (SLS) and Dynamic Light Scattering (DLS) simultaneously using the same sample conditions as for the second AUC study. Correlation curves and hydrodynamic radius $\left(R_{\mathrm{H}}\right)$ distributions from DLS show the presence of one contribution for most of the samples (example in Figure S3). Larger species $\left(R_{\mathrm{H}} \approx 100 \mathrm{~nm}\right)$ were marginally detected in negligible amounts of the peak intensity and are considered as irrelevant dust.

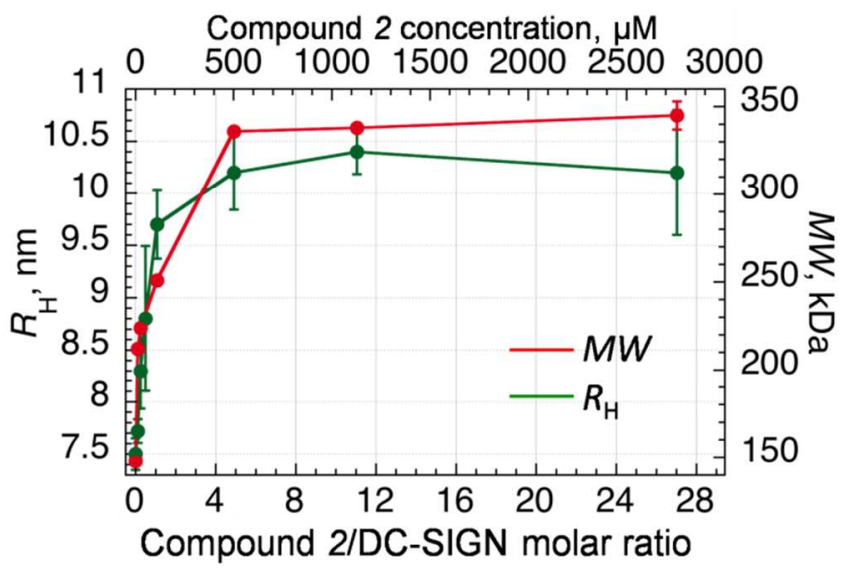

Figure 6. Results of static and dynamic light scattering of $102 \mu \mathrm{M}$ DC-SIGN ECD alone and in presence of 2 at different 2/DC-SIGN molar ratios. The error bars represent standard deviation values.

The $R_{\mathrm{H}}$ and $M W$ values were plotted against the 2/DC-SIGN molar ratios (Figure 6). SLS and DLS do not allow distinguishing monomers and dimers of tetrameric DC-SIGN ECD. The measured $M W$ and $R_{\mathrm{H}}$ are mean values dominated by the larger species, since scattered intensity is proportional to $\sum c_{\mathrm{i}} M W_{\mathrm{i}}^{2}, c_{\mathrm{i}}$ and $M W_{\mathrm{i}}$ being the weight concentration and molar mass of species $i$. When increasing the 2/DC-SIGN ECD ratio, $M W$ increases from $148 \mathrm{kDa}$ for DC-SIGN ECD alone and reaches a constant value of $350 \mathrm{kDa}$ at ratio 5 . Correspondingly, $R_{\mathrm{H}}$ increases from $7.5 \mathrm{~nm}$ without 2 to a maximum value of $10.4 \mathrm{~nm}$ at ratio 11 , followed by a slight and perhaps irrelevant decrease to $10.2 \mathrm{~nm}$ at ratio 27 . The evolution of $M W$ and $R_{\mathrm{H}}$ reflects the general behavior observed in AUC (Figure 5B). We note that in 
the absence of 2, $M W$ from SLS is close to the theoretical value for a tetrameric DC-SIGN ECD $(154.827 \mathrm{kDa}) ; R_{\mathrm{H}}$ is close to the value of $7.7 \mathrm{~nm}$ obtained from the combination of $s_{20 \mathrm{w}}=4.8 \mathrm{~S}$ and the theoretical $M W$ of a tetramer. The maximum $M W$-value from SLS (350 $\mathrm{kDa})$ is close to the expected value for a dimer of tetramers $(309.654 \mathrm{kDa})$, while the combination of $s_{20 \mathrm{w}}=6.3 \mathrm{~S}$ with $M W=309.654 \mathrm{kDa}$ gives $R_{\mathrm{H}}=11.7 \mathrm{~nm}$, close to the experimental value of 10.4 from DLS above 2/DCSIGN ratio $\geq 5$.

Altogether, AUC, DLS and SLS data support that 2 promotes the dimerization of DC-SIGN tetramers when 2/DC-SIGN ECD ratios are between 1 and 5, while higher excess of 2 saturates all the binding sites, which interrupts the further bridging.

In order to get an insight to the possible organization of compound 2-mediated DC-SIGN assembly, we generated a model of a supramolecular complex where two DC-SIGN ECDs were bridged by four compounds 2 using a symmetry docking algorithm(25). The best resulting model apparently showed no steric clashes between both facing DC-SIGN ECD (Figure 7). Finally, the constructed model was subjected to HydroPro calculations(26), and yielded the theoretical $s$ value for the model structure of $6.8 \mathrm{~S}$, which in turn gives $R_{\mathrm{H}}$ value of $9.6 \mathrm{~nm}$. This $R_{\mathrm{H}}$ value is in agreement, within standard deviation, with the experimentally measured $R_{\mathrm{H}}$ maximum (Figure 6). This suggests that the supra-molecular assembly is compatible with such an elongated conformation. However, this model must be taken only as a rough approximation of the real situation: it must be kept in mind that such a system is presumably rather dynamic due to relatively low affinity of single mannose residue, and thus possibly adopts different conformations and/or a mix of species in solution totally loaded with 2 (Figure 7) or partially bridged by one, two or three molecules of compound 2. 


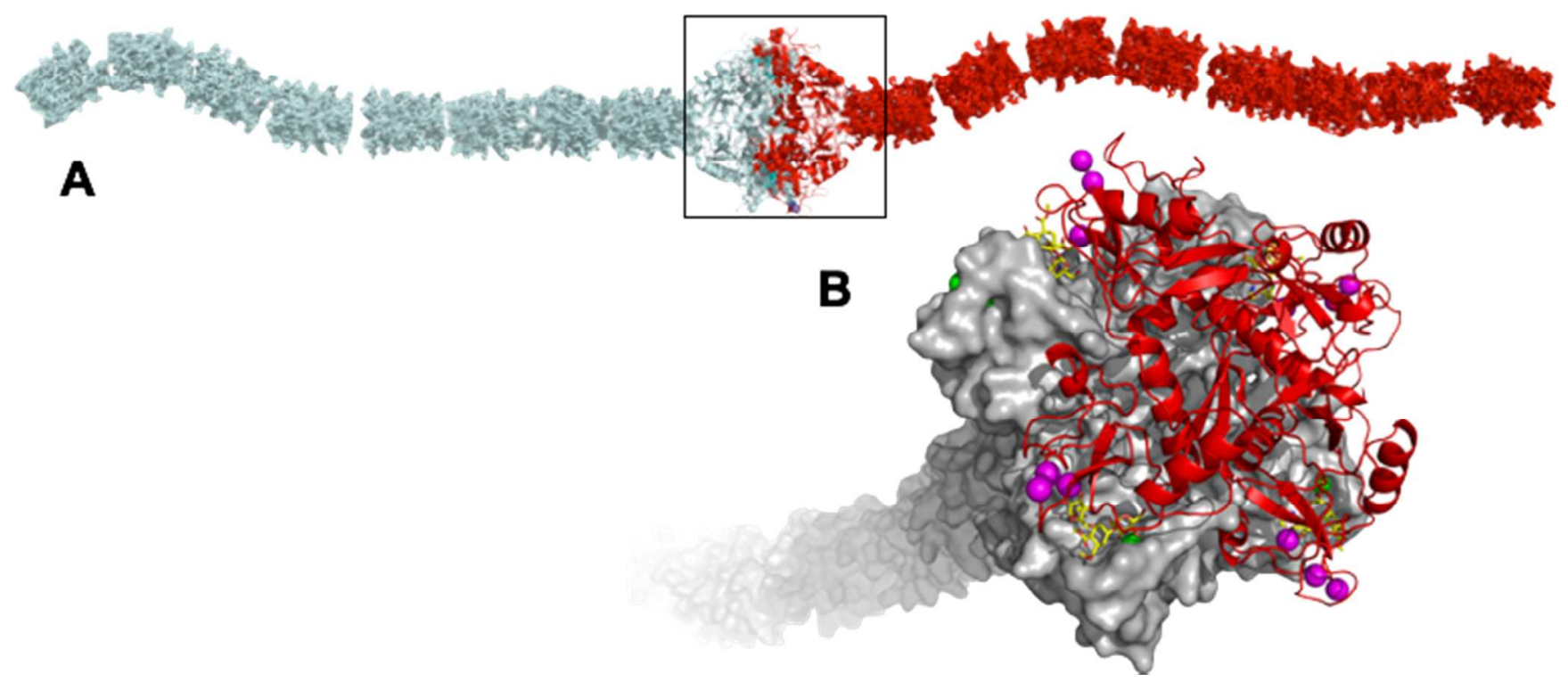

Figure 7. A proposed model of the bridging of DC-SIGN tetramers by four molecules of compound 2. (A) Overall organization of bridged DC-SIGN ECD. (B) Close-up view of the interaction area marked by a black frame in panel A. The color scheme: one of the two DC-SIGN tetramers is represented as a gray surface, and the other one as red cartoon. For clarity the neck domain of the red DC-SIGN ECD is omitted. Carbons of $\mathbf{2}$ are yellow; oxygens and nitrogens are red and blue; $\mathrm{Ca}^{2+}$ ions are green or pink spheres for the gray and red DC-SIGN tetramers, respectively.

\section{Comparison of the ability of multivalent systems bearing 1 or 2 to inhibit HIV trans-infection}

In our previous study, due to the better activity of $\mathbf{2}$ observed in SPR competition assays, only this molecule was used to generate a tetravalent dendron which was tested with good results in cellular studies of HIV trans-infection inhibition(17). Upon full characterization of the binding modes of 1 and 2 and after the results described in this paper, inhibition of HIV-1 trans-infection was readdressed in the same cellular model using both 1 and 2-based tetravalent dendrons (3.1 and 3.2) in order to compare their HIV blocking abilities. Additionally, 32-valent dendrimers bearing 1 or 2 (Figure 1A) were also studied. The results showed that both dendrons were able to reduce HIV transmission to $\mathrm{CD}^{+} \mathrm{T}$-cells by a very similar extent (Figure 8 ): $5 \mu \mathrm{M}$ concentrations displayed $\sim 50 \%$ infection inhibition, while incubation with $50 \mu \mathrm{M}$ dendrons reduced infection almost to $90 \%$.

Due to the higher binding avidity, the dendrimers with both $\mathbf{1}$ and $\mathbf{2}$ displayed significantly higher efficiencies than dendrons and almost completely inhibited the infection at $50 \mu \mathrm{M}$ concentration. 


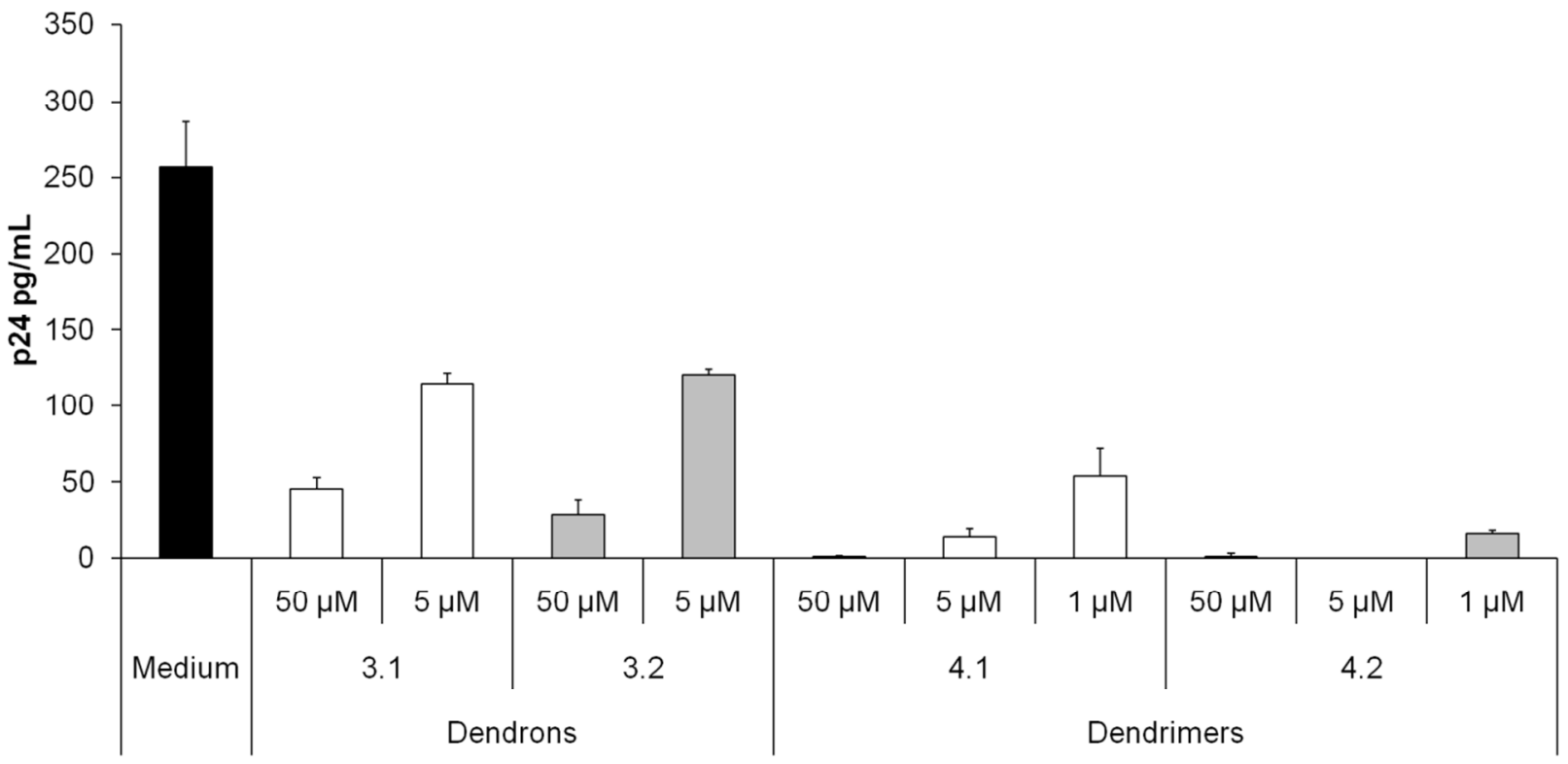

Figure 8. Comparison of HIV trans-infection inhibition by multivalent systems 3.1, 3.2, 4.1 and 4.2. HIV infection is expressed in terms of $\mathrm{p} 24$ concentration $(m e a n \pm \mathrm{SD})$. Data obtained in duplicate, from three healthy donors.

Despite slight differences of inhibitory efficiencies of $\mathbf{1}$ and $\mathbf{2}$ bearing dendrimers at lower concentrations, these data correlate well with above described results and confirm once again that both pseudosaccharides have very similar activities once they are tethered to multivalent scaffolds. It definitely confirms that $\mathbf{1}$ is comparable to $\mathbf{2}$ and any residual difference is easily offset by the superior synthetic accessibility of $\mathbf{1}$, which lends itself to easier optimization.

In summary, the development of multivalent glycomimetic antagonists required to efficiently compete with natural multivalent interactions(27) is a multistep process comprising the design of active monovalent ligands, screening for the monovalent leads, and their presentation on the designed multivalent scaffolds. Here we demonstrated the importance of a thorough and critical evaluation of the monovalent ligands, a crucial step for the rational design of the lectin antagonists where the choice of a false positive could cost void efforts to develop a final active multivalent compound. We have designed small related pseudosaccharides $\mathbf{1}$ and $\mathbf{2}$ as starting monovalent compounds. Although at monovalent level compound $\mathbf{2}$ had markedly better activity than 1, it was lost at the multivalent presentation resulting in comparable activities of multivalent forms of $\mathbf{1}$ and $\mathbf{2}$. Further structural investigation of DC-SIGN interaction with $\mathbf{2}$ has revealed an unexpected property of this small molecule to induce DCSIGN ECD dimerization. Interaction of oligomannosides with C-lectins, including DC-SIGN, is indeed a dynamic process, which allows multiple binding modes using different mannose residues at the primary $\mathrm{Ca}^{2+}$ site. Hence, it is not surprising that $\mathbf{2}$ can engage the protein with both of its sugar moieties. However, although bridging lectin-carbohydrate interactions are common and have a 
biological relevance $(28,29)$, the case presented here is unique, considering the small size of the molecule and the low affinity of single mannose residues for DC-SIGN. Probably, the absence of steric hindrance between both DC-SIGN ECDs in the complex, as suggested by the modeling, allows such bridging to occur. In biological systems the most common situation of multivalent sugar/protein interactions is the formation of carbohydrate-lectin lattices(30) and lectin bridging property of glycomimetic molecules has been previously engineered in relatively large multimeric presentations(31-34). However, to the best of our knowledge, bridging effects by an oligosaccharide as small as a (pseudo)trimer have never been observed with one exception in the particular case of crystallization conditions(33).

Unfortunately, this property of $\mathbf{2}$ depends on a precise format of the interaction assay and requires both ligand and protein to be simultaneously in solution. This doesn't correspond to the biologically relevant situation where the receptor is anchored to cell membrane and the ligand in solution. Additionally, the bridging ability of $\mathbf{2}$ apparently is lost, even in solution assays, when it is tethered to a multivalent scaffold. Thus this ligand cannot fully profit of avidity effects generated by multivalent presentations. In our development of DC-SIGN inhibitors, where we aim to design multivalent platforms bearing multiple copies of moderately active glycomimetic leads, the bridging ability of $\mathbf{2}$ was the underlying reason for its overestimation in competition assays. Once this ligand is tethered to multivalent scaffolds, the second mannose moiety acts basically as an extended linker providing no advantages. Without a thorough structural characterization of the binding mode of 1 and 2 to DC-SIGN (reference (20) and this work, respectively), compound 2 would have been chosen for further chemical optimization on the sole basis of $\mathrm{IC}_{50}$ results. This option would have been extremely costly, on a synthetic point of view, and probably ineffective at the end. From the outcome of these interaction analyses, it is clear that optimization of the monovalent ligand, therefore, should focus on the structure of the pseudo-disaccharide 1, which is synthetically much simpler than 2 . Our recent improvements in this direction have been recently reported(35). Besides providing a structural rationale for observations made during our DC-SIGN antagonists research program, these results deliver a general lesson for the design of lectin antagonists.

\section{Methods}

Compound synthesis. 
Syntheses of 1(36), 2, 3.2(17) 3.1, 4.1 and 4.2(19) were described previously.

\section{DC-SIGN production and purification}

DC-SIGN CRD-StrepTagII (S-CRD) and ECD constructs were produced as described previously(10, 20).

\section{DC-SIGN/2 co-crystallization, data collection and structure solution}

Crystallization was performed at $20^{\circ} \mathrm{C}$ in EasyXtal plates (Qiagen) by hanging-drop vapor-diffusion method. The drops were prepared combining $1 \mu \mathrm{L}$ of a purified DC-SIGN S-CRD $\left(4.4 \mathrm{mg} \mathrm{mL}^{-1}\right.$ in $25 \mathrm{mM}$ Tris- $\mathrm{HCl} \mathrm{pH} \mathrm{8,} 150 \mathrm{mM} \mathrm{NaCl}, 4 \mathrm{mM} \mathrm{CaCl}_{2}$, buffer-A) and 2 (300 mM) mixture (9:1, v/v) with $1 \mu \mathrm{L}$ of reservoir solution and equilibrated against $1 \mathrm{~mL}$ of reservoir solution. The best crystals were obtained when reservoir solution was 35\% PEG3350, $100 \mathrm{mM}$ cacodylate $\mathrm{pH} 6.5$, and $200 \mathrm{mM} \mathrm{NaCl}$. The X-ray diffraction data collection, structure solution and refinement are described in Supplementary Information. Crystal structure of DC-SIGN CRD/2 has been deposited in Protein Data Bank under PDB code $2 \times r 6$.

\section{Isothermal titration microcalorimetry}

ITC experiments were performed at $25^{\circ} \mathrm{C}$ using Microcal/VP-ITC microcalorimeter (Microcal, Northampton, MA) with $1.4 \mathrm{~mL}$ cell volume. . The pseudosaccharides and DC-SIGN ECD were prepared in buffer-A. Pseudosaccharides were stepwise injected (10 $\mu \mathrm{L}$ aliquots) to DC-SIGN solution using 5 min intervals between injections. In the first set of experiments $71 \mu \mathrm{M}$ (in terms of binding sites) lectin and $12.7 \mathrm{mM}$ pseudosaccharide concentrations were used; in the second set $1.18 \mathrm{mM} 2$ was titrated to $214 \mu \mathrm{M}$ DC-SIGN ECD. The blank titrations (compounds to buffer) were done for subtraction of dilution heat from the integrated data. A one-site binding model was fit to the data (Microcal Origin-7) yielding association constants $\left(K_{\mathrm{A}}\right)$ and binding enthalpies $(\Delta H)$. The free energy changes $(\Delta G)$ and entropy $(\Delta S)$ were calculated using equation:

$$
\Delta G=\Delta H-T \Delta S=-R T \ln K_{\mathrm{A}}
$$

where $T$ is the absolute temperature, and $R=8.314 \mathrm{~J} \mathrm{~mol}^{-1} \mathrm{~K}^{-1}$.

\section{Static and Dynamic Light Scattering}

The SLS and DLS were performed using DynaPro/Nanostar instrument (Wyatt Technology Corp., Santa Barbara, USA) equipped with $658 \mathrm{~nm}$ laser at $90^{\circ}$ scattering angle. Triplicate measurements of 
10 scattering readings per sample in a quartz cuvette were recorded at $25^{\circ} \mathrm{C}$. The samples, prepared in buffer-A and centrifuged prior to analysis, contained DC-SIGN ECD alone $(102 \mu \mathrm{M}$ with respect to binding sites) or with 2 at different 2/DC-SIGN molar ratios $(0,0.11,0.25,0.5,1,5,11$ and 27). The data were analyzed with Dynamics-7.1 software (Wyatt Technology Corp.).

\section{Analytical Ultracentrifugation}

SV experiments were performed using Beckman XL-I analytical ultracentrifuge with AN-50 TI rotor (Beckman instruments), at $20^{\circ} \mathrm{C}$ and $42000 \mathrm{rpm}$, using $55 \mu \mathrm{l}$ samples, loaded into the two-channel $0.15 \mathrm{~cm}$ path-length centerpieces with sapphire windows (Nanolytics). The absorbance at $280 \mathrm{~nm}$ was monitored every 5 or $11 \mathrm{~min}$ for the first and second set experiments, respectively, with a $30 \mu \mathrm{m}$ radial step size.

The samples were prepared in buffer-A. The first set of experiments was done with samples retrieved from first set ITC measurements, thus contained DC-SIGN ECD alone at $62 \mu \mathrm{M}$, or with $1.63 \mathrm{mM} 1$ or 2. The identical freshly prepared samples were used for the control, giving identical results.

For the second set, the samples contained $102 \mu \mathrm{M}$ DC-SIGN ECD alone or with 2 added at different 2/DC-SIGN molar ratios $(0,0.25,0.5,1,5,11$ and 27). The MW and partial specific volume $(\bar{v})$ of DC-SIGN tetramers were estimated from amino acid composition using SEDNTERP software and resulted $154827 \mathrm{Da}$ and $0.733 \mathrm{~cm}^{3} \mathrm{~g}^{-1}$, respectively. The $\bar{v}$-values for $\mathbf{1}$ and $\mathbf{2}$ were considered equal to $\bar{v}$-value of a hexose $\left(0.607 \mathrm{~cm}^{3} \mathrm{~g}^{-1}\right)$. SV profiles were analyzed using the size distribution analysis(39) embedded in the SEDFIT software (available at http://www.analyticalultracentrifugation.com), and for each analysis by global modeling taking typically 20 regularly spaced experimental profiles obtained over $4 \mathrm{~h}$. The $c(s)$ analysis was performed considering 200 particles, and fitting the frictional ratio, $f / f_{0}$. For the regularization, a 0.68 confidence level was used. All $s$-values were corrected for solvent density and viscosity, and thus are given as $s_{20 \mathrm{w}}$-values.

\section{DC-SIGN/2 bridging complex modeling.}

To generate a tetrahedral DC-SIGN assembly, we used a novel symmetry docking algorithm(25). We assembled a tetrahedral octamer possessing D4 symmetry starting from a monomer of the reported crystal structure (PDB 2xr6) with a single mannose in the $\mathrm{Ca}^{2+}$ binding-site using the exhaustive search in six degrees of freedom. Then, we selected an octameric solution that fulfilled geometric constraints at the coiled-coil start and fitted the pseudotrisaccharide 2 into its binding pocket using Autodock/Vina 
software(40). Solutions, where ECD organization was compatible with the previously described DCSIGN ECD SAXS envelope, have been conserved(10). To set-up the ligand docking simulations, we used Autodock/Vina plugin for PyMOL(41).

\section{Calculation of theoretical hydrodynamic radius from the model.}

HYDROPRO program(26) was used to obtain the theoretical sedimentation coefficient value for the model of bridged DC-SIGN tetramers. The experimental values of solvent viscosity, density and temperature were $\eta=0.01024$ poise, $\rho=1.006 \mathrm{~g} \mathrm{~cm}^{-3}$, and the temperature was $293 \mathrm{~K}$. The resulting sedimentation coefficient $s$-value of $6.848 \mathrm{~S}$ was used to calculate theoretical $R_{\mathrm{H}}$ using Svedberg's equation:

$$
s=\frac{M W \cdot(1-\rho \cdot \bar{v})}{N_{A} \cdot 6 \cdot \pi \cdot \eta \cdot R_{H}},
$$

where $M W$ the molecular mass of the dimer of DC-SIGN tetramers (309.654 kDa), and $\bar{v}$ is the partial specific volume of the dimer of tetramers $\left(0.733 \mathrm{~cm}^{3} \mathrm{~g}^{-1}\right)$.

\section{Infection studies.}

The studies of HIV trans-infection inhibition by 3.1, 3.2, 4.1 and 4.2 without removal of compounds prior to virus exposure were performed as described previously(17).

\section{Acknowledgments:}

This work was supported by the Marie Curie ITN FP7 project CARMUSYS (PITN-GA-2008-213592) and the Agence Nationale de la Recherche (ANR-11-MONU-006-01). A. Berzi was supported by a fellowship of UNIMI and Regione Lombardia (Programma "Dote Ricerca- Rafforzare il capitale umano", POR-Ob.2 Asse IV-FSE 2007-2013). We are grateful to Pr. Anne Imberty and Dr. Aymeric Audfray for an access to ITC instrument and for their kind support and advice. FF thanks the Institut Universitaire de France for financial support. This work has been conducted thanks to the access to the MP3, AUC and PAOL platforms of the Partnership for Structural Biology and the Institut de Biologie Structurale in Grenoble (PSB/IBS).

\section{References}

1. Banchereau, J., and Steinman, R. M. (1998) Dendritic cells and the control of immunity, Nature 392, 245-252.

2. Figdor, C. G., van Kooyk, Y., and Adema, G. J. (2002) C-type lectin receptors on dendritic cells and Langerhans cells, Nat. Rev. Immunol. 2, 77-84.

3. Geijtenbeek, T. B., van Vliet, S. J., Engering, A., t Hart, B. A., and van Kooyk, Y. (2004) 
Self- and nonself-recognition by C-type lectins on dendritic cells, Annu. Rev. Immunol. 22, 33-54.

4. Geijtenbeek, T. B., Kwon, D. S., Torensma, R., van Vliet, S. J., van Duijnhoven, G. C., Middel, J., Cornelissen, I. L., Nottet, H. S., KewalRamani, V. N., Littman, D. R., Figdor, C. G., and van Kooyk, Y. (2000) DC-SIGN, a dendritic cell-specific HIV-1-binding protein that enhances trans-infection of T cells, Cell 100, 587-597.

5. Svajger, U., Anderluh, M., Jeras, M., and Obermajer, N. (2010) C-type lectin DC-SIGN: an adhesion, signalling and antigen-uptake molecule that guides dendritic cells in immunity., Cell. Signal. 22, 1397-1405.

6. van Kooyk, Y., and Geijtenbeek, T. B. (2003) DC-SIGN: escape mechanism for pathogens, Nat Rev Immunol 3, 697-709.

7. Geijtenbeek, T. B. H., Dunnen, den, J., and Gringhuis, S. I. (2009) Pathogen recognition by DC-SIGN shapes adaptive immunity., Future Microbiol 4, 879-890.

8. Feinberg, H., Mitchell, D. A., Drickamer, K., and Weis, W. I. (2001) Structural basis for selective recognition of oligosaccharides by DC-SIGN and DC-SIGNR, Science 294, 2163-2166.

9. Guo, Y., Feinberg, H., Conroy, E., Mitchell, D. A., Alvarez, R., Blixt, O., Taylor, M. E., Weis, W. I., and Drickamer, K. (2004) Structural basis for distinct ligand-binding and targeting properties of the receptors DC-SIGN and DC-SIGNR., Nat Struct Mol Biol 11, 591-598.

10. Tabarani, G., Thépaut, M., Stroebel, D., Ebel, C., Vivès, C., Vachette, P., Durand, D., and Fieschi, F. (2009) DC-SIGN neck domain is a pH-sensor controlling oligomerization: SAXS and hydrodynamic studies of extracellular domain., J Biol Chem 284, 2122921240.

11. Cambi, A., de Lange, F., van Maarseveen, N. M., Nijhuis, M., Joosten, B., van Dijk, E. M., de Bakker, B. I., Fransen, J. A., Bovee-Geurts, P. H., van Leeuwen, F. N., Van Hulst, N. F., and Figdor, C. G. (2004) Microdomains of the C-type lectin DC-SIGN are portals for virus entry into dendritic cells, J Cell Biol 164, 145-155.

12. de Bakker, B. I., de Lange, F., Cambi, A., Korterik, J. P., van Dijk, E. M., Van Hulst, N. F., Figdor, C. G., and Garcia-Parajo, M. F. (2007) Nanoscale organization of the pathogen receptor DC-SIGN mapped by single-molecule high-resolution fluorescence microscopy, Chemphyschem 8, 1473-1480.

13. Reina, J., Bernardi, A., Clerici, M., and Rojo, J. (2010) HIV microbicides: state-of-the-art and new perspectives on the development of entry inhibitors, Future Med. Chem. 2, 11411159.

14. Anderluh, M., Jug, G., Svajger, U., and Obermajer, N. (2012) DC-SIGN antagonists, a potential new class of anti-infectives., Curr. Med. Chem. 19, 992-1007.

15. Andreini, M., Doknic, D., Sutkeviciute, I., Reina, J. J., Duan, J., Chabrol, E., Thépaut, M., Moroni, E., Doro, F., Belvisi, L., Weiser, J., Rojo, J., Fieschi, F., and Bernardi, A. (2011) Second generation of fucose-based DC-SIGN ligands : affinity improvement and specificity versus Langerin., Org Biomol Chem 9, 5778-5786.

16. de Witte, L., Nabatov, A., Pion, M., Fluitsma, D., de Jong, M. A. W. P., de Gruijl, T., Piguet, V., van Kooyk, Y., and Geijtenbeek, T. B. H. (2007) Langerin is a natural barrier to HIV-1 transmission by Langerhans cells, Nat Med 13, 367-371.

17. Sattin, S., Daghetti, A., Thépaut, M., Berzi, A., Sánchez-Navarro, M., Tabarani, G., Rojo, J., Fieschi, F., (null), and Bernardi, A. (2010) Inhibition of DC-SIGN-mediated HIV infection by a linear trimannoside mimic in a tetravalent presentation., ACS Chem Biol 5, 301-312. 
18. Berzi, A., Reina, J. J., Ottria, R., Sutkeviciute, I., Antonazzo, P., Sánchez-Navarro, M., Chabrol, E., (null), (null), Cetin, I., Rojo, J., Fieschi, F., Bernardi, A., and Clerici, M. (2012) A glycomimetic compound inhibits DC-SIGN-mediated HIV infection in cellular and cervical explant models., AIDS 26, 127-137.

19. Luczkowiak, J., Sattin, S., Sutkeviciute, I., Reina, J. J., Sánchez-Navarro, M., Thépaut, M., Martinez-Prats, L., Daghetti, A., Fieschi, F., Delgado, R., Bernardi, A., and Rojo, J. (2011) Pseudosaccharide Functionalized Dendrimers as Potent Inhibitors of DC-SIGN Dependent Ebola Pseudotyped Viral Infection., Bioconjug Chem 22, 1354-1365.

20. Thépaut, M., Guzzi, C., Sutkeviciute, I., Sattin, S., Ribeiro-Viana, R., Varga, N., Chabrol, E., Rojo, J., Bernardi, A., Angulo, J., Nieto, P. M., and Fieschi, F. (2013) Structure of a Glycomimetic Ligand in the Carbohydrate Recognition Domain of C-type Lectin DCSIGN. Structural Requirements for Selectivity and Ligand Design., J Am Chem Soc 135, 2518-2529.

21. Turnbull, W. B., and Daranas, A. H. (2003) On the value of c: can low affinity systems be studied by isothermal titration calorimetry?, J Am Chem Soc 125, 14859-14866.

22. Tabarani, G., Reina, J. J., Ebel, C., Vivès, C., Lortat-Jacob, H., Rojo, J., and Fieschi, F. (2006) Mannose hyperbranched dendritic polymers interact with clustered organization of DC-SIGN and inhibit gp120 binding., FEBS Lett 580, 2402-2408.

23. Schuck, P. (2003) On the analysis of protein self-association by sedimentation velocity analytical ultracentrifugation, Anal Biochem 320, 104-124.

24. Zhao, H., Balbo, A., Brown, P. H., and Schuck, P. (2011) The boundary structure in the analysis of reversibly interacting systems by sedimentation velocity., Methods 54, 16-30.

25. Ritchie, D. W., and Grudinin, S. Some recent progress on generating symmetric protein complexes using spherical polar Fourier docking correlations. In Proceedings of the 14th Open Days in Biology, Computer Science and Mathematics (JOBIM), July 1-4, 2013, Toulouse (France).

26. Garcia De La Torre, J., Huertas, M. L., and Carrasco, B. (2000) Calculation of hydrodynamic properties of globular proteins from their atomic-level structure, Biophys $J$ 78, 719-730.

27. Bernardi, A., Jiménez-Barbero, J., Casnati, A., De Castro, C., Darbre, T., Fieschi, F., Finne, J., Funken, H., Jaeger, K.-E., Lahmann, M., Lindhorst, T. K., Marradi, M., Messner, P., Molinaro, A., Murphy, P. V., Nativi, C., Oscarson, S., Penadés, S., Peri, F., Pieters, R. J., Renaudet, O., Reymond, J.-L., Richichi, B., Rojo, J., Sansone, F., Schäffer, C., Turnbull, W. B., Velasco-Torrijos, T., Vidal, S., Vincent, S., Wennekes, T., Zuilhof, H., and Imberty, A. (2013) Multivalent glycoconjugates as anti-pathogenic agents, Chem. Soc. Rev. 42, 4709-4727.

28. Dam, T. K., Brewer, C. F., and Brewer, F. C. (2010) Maintenance of cell surface glycan density by lectin-glycan interactions: a homeostatic and innate immune regulatory mechanism., Glycobiology 20, 1061-1064.

29. Dam, T. K., and Brewer, C. F. (2008) Effects of clustered epitopes in multivalent ligandreceptor interactions., Biochemistry 47, 8470-8476.

30. Fred Brewer, C. (2002) Binding and cross-linking properties of galectins., Biochim Biophys Acta 1572, 255-262.

31. Gestwicki, J. E., Cairo, C. W., Strong, L. E., Oetjen, K. A., and Kiessling, L. L. (2002) Influencing receptor-ligand binding mechanisms with multivalent ligand architecture., $J$ Am Chem Soc 124, 14922-14933.

32. Sisu, C., Baron, A. J., Branderhorst, H. M., Connell, S. D., Weijers, C. A. G. M., De Vries, R., Hayes, E. D., Pukin, A. V., Gilbert, M., Pieters, R. J., Zuilhof, H., Visser, G. M., and 
Turnbull, W. B. (2009) The Influence of Ligand Valency on Aggregation Mechanisms for Inhibiting Bacterial Toxins, ChemBioChem 10, 329-337.

33. Lameignere, E., Shiao, T. C., Roy, R., Wimmerova, M., Dubreuil, F., Varrot, A., and Imberty, A. (2010) Structural basis of the affinity for oligomannosides and analogs displayed by BC2L-A, a Burkholderia cenocepacia soluble lectin., Glycobiology 20, 8798.

34. Wittmann, V., and Pieters, R. J. (2013) Bridging lectin binding sites by multivalent carbohydrates., Chem. Soc. Rev. 42, 4492-4503.

35. Varga, N., Sutkeviciute, I., Guzzi, C., McGeagh, J., Petit-Haertlein, I., Gugliotta, S., Weiser, J., Angulo, J., Fieschi, F., and Bernardi, A. (2013) Selective targeting of dendritic cell-specific intercellular adhesion molecule-3-grabbing nonintegrin (DC-SIGN) with mannose-based glycomimetics: synthesis and interaction studies of bis(benzylamide) derivatives of a pseudomannobioside., Chemistry (Weinheim an der Bergstrasse, Germany) 19, 4786-4797.

36. Reina, J. J., Sattin, S., Invernizzi, D., Mari, S., Martinez-Prats, L., Tabarani, G., Fieschi, F., Delgado, R., Nieto, P. M., Rojo, J., and Bernardi, A. (2007) 1,2-Mannobioside mimic: synthesis, DC-SIGN interaction by NMR and docking, and antiviral activity., ChemMedChem 2, 1030-1036.

37. Emsley, P., and Cowtan, K. (2004) Coot: model-building tools for molecular graphics, Acta Crystallogr D Biol Crystallogr 60, 2126-2132.

38. Collaborative Computational Project, N. 4. (1994) The CCP4 suite: programs for protein crystallography ., Acta Crystallogr. D 50, 760-763.

39. Schuck, P. (2000) Size-distribution analysis of macromolecules by sedimentation velocity ultracentrifugation and lamm equation modeling, Biophys $J$ 78, 1606-1619.

40. Trott, O., and Olson, A. J. (2010) AutoDock Vina: improving the speed and accuracy of docking with a new scoring function, efficient optimization, and multithreading, $J$ Comput Chem 31, 455-461.

41. Seeliger, D., and Groot, B. L. (2010) Ligand docking and binding site analysis with PyMOL and Autodock/Vina, J. Comput. Aided Mol. Des. 24, 417-422. 


\section{Table of Contents Graphics}

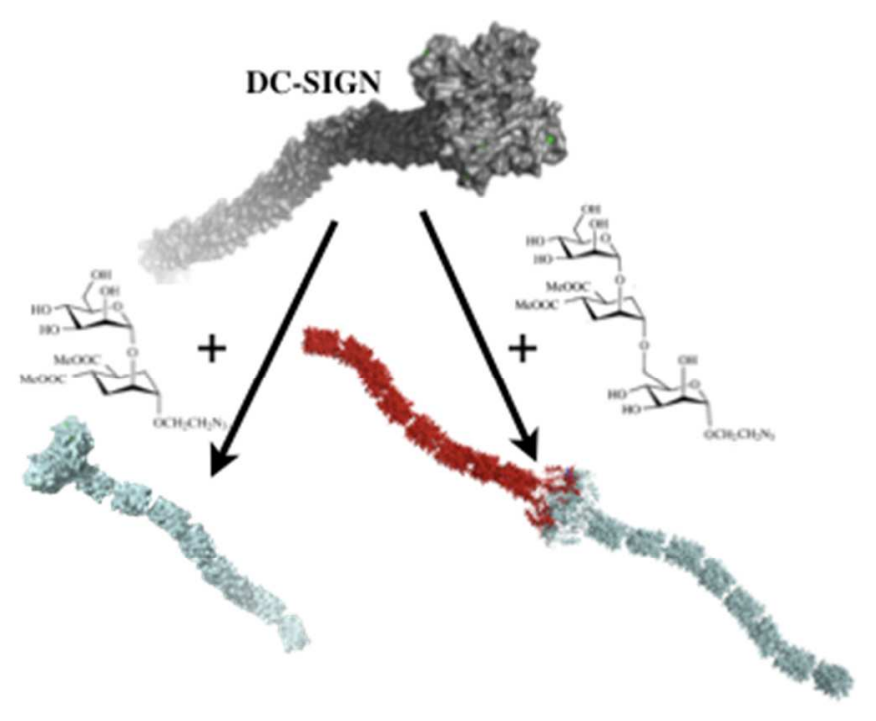

22

23

25

26

27

28

29

30

31

32

33

34

35

36

37

38

39

40

41

42

43

44

45

46

47

48

49

50

51

52

53

54

55

56

57

58

59

60 